Article

\title{
Research on Steady States of Fuzzy Cognitive Map and its Application in Three-Rivers Ecosystem
}

\author{
Zhen Peng ${ }^{1, *}$, Lifeng $\mathrm{Wu}^{2}$ and Zhenguo Chen ${ }^{3}$ \\ Received: 13 October 2015; Accepted: 29 December 2015; Published: 4 January 2016 \\ Academic Editors: Nikos E. Mastorakis and Marc A. Rosen \\ 1 Information Management Department, Beijing Institute of Petrochemical Technology, Beijing 102617, China \\ 2 College of Information Engineering, Capital Normal University, Beijing 100048, China; wooleef@gmail.com \\ 3 Computer Department, North China Institute of Science and Technology, East Yanjiao, Beijing 101601, China; \\ 6638752@163.com \\ * Correspondence: zhenpeng@bipt.edu.cn; Tel.: +86-10-6022-8013
}

\begin{abstract}
Fuzzy Cognitive Map (FCM) offers many advantages such intuitive knowledge representation and fast numerical reasoning ability, etc. It suits modeling and decision-making of dynamic systems. With the aims to effectively help to analyze and control system sustainable evolution, the paper defines the steady states of fixed point and limited cycle of a FCM modeling system. Accordingly, the rules of steady states of the FCM model and the factors influencing the steady states are presented and proved. The Three-Rivers represents a system including population, ecological environment, social development and their relationships. Based on the relationships, the Three-Rivers ecosystem is modeled by FCM and the Three-Rivers ecosystemsustainable evolutionis analyzed bythe rules of the steady states of FCM.
\end{abstract}

Keywords: Fuzzy Cognitive Map (FCM); fixed point; limited cycle; Three-Rivers ecosystem; sustainable evolution

\section{Introduction}

At present, there is an urgent need for a kind of intelligent tool to model dynamic systems for analysis and control. Fuzzy Cognitive Map (FCM) [1,2], as a soft computing tool, was proposed by Kosko in 1986 on the basis of Axelord's Cognitive Map(CM). FCM extends the ternary logical relationship into the interval $[-1,1]$. The most attractive characteristics of FCM are the flexibility of the system model, the comprehensive operation and the abstractive representation of system. Over the last several years, a variety of FCMs have been used for representing knowledge and artificial intelligence in engineering applications, for instance infault detection [3], geographical information systems [4], social system modeling [5], medical decision-making [6], individual behaviors simulation [7], environmental management [8], etc.

There are three parts or three functions in the modeling of FCM. The first part [9-11] is to simulate a specific system by some learning the algorithms of FCM such as NHL (Nonlinear Hebbian Learning) andRCGA (Real-Coded Genetic Algorithm), where the association weight of FCM needs to be established by data resources. They emphasize the determination of the relationships of concepts in the system. The second part [12-15] is used for system evaluation by the FCM inference mechanism, which focuses on the use of interactions between different concepts. It has the purpose of system evaluation according to FCM inference. The third part [16-18] is the state transformation of FCM, where the transforming rule and the transforming process of steady state are focused on. They are used to analyze and control system evolution.

In the above-mentioned third part, there issome research onthe view of steady states of FCM. For example, some statements in the paper [16] are inaccurate, such as where it holds that the length of the 
limited cycle should be less than the number of concepts or otherwise cross-talk can occur. However, it does not analyze and prove the transforming rules of steady states (fixed point and limited cycle) of the FCM model at all. Thus, in order to further analyze the evolution of the system fundamentally, some definitions and proofs are researched and proposed in the paper, which are used to analyze system evolution changes and influence factors. Additionally, they are applied in the evolution analysis of the Three-Rivers ecosystem. The Three-Rivers system is modeled by FCM from the three sections of population, ecological environment, social development and their relationshipsat themacro-level. The evolutions of the Three-Rivers ecosystem are studied by the steady states of FCM from the macro-level for sustainable development decisions.

The outline of this paper follows. The introduction presents related work for FCM and the purpose of the study. In Section 2, FCM including the inference mechanism and the steady states and the related concepts about the steady states of FCM are well described and established. Section 3 defines and proves the rules of two steady states. Section 4 is an application of the rules in the Three-Rivers ecosystem and discusses system evolution in different states. The final section briefly concludes the paper.

\section{FCM}

The structure of FCM is a 4-tuple $(C, E, W, A)$.

$C=\left\{C_{1}, C_{2}, \ldots, C_{n}\right\}$ is a set of concept nodes representing objects in the real system.

$E=\left\{\left\langle C_{i}, C_{j}>\right| C_{i}, C_{j} \in C\right\}$ is oriented arcs denoting the causality relationship between two objects in the system.

$W=\left\{w_{i j} \mid w_{i j}\right.$ is the weight value of the interconnection $\left\langle C_{i}, C_{j}>\right\} . W$ is a square matrix of $n \times n$ showing the association weight map between objects. The $w_{i j}$ belongs to the interval $[-1,1]$ representing the fuzzy degree between concepts $C_{i}$ and $C_{j}$.

- If there is positive causality between concepts $C_{i}$ and $C_{j}, w_{i j}>0$. An increase of the value of concept $C_{i}$ will cause an increase of the value of concept $C_{j}$. A decrease of the value of $C_{i}$ will lead to a decrease of the value of $C_{j}$.

- If there is inverse causality between the two concepts, $w_{i j}<0$. An increase of the value of concept $C_{i}$ will cause a decrease of the value of the second concept. A decrease of the value of concept $C_{i}$ will cause an increase of the value of the concept $C_{j}$.

- If there is no relationship between the two concepts, $w_{i j}=0$.

For example, an FCM on public health and its weight matrix are shown in Figures 1 and 2.

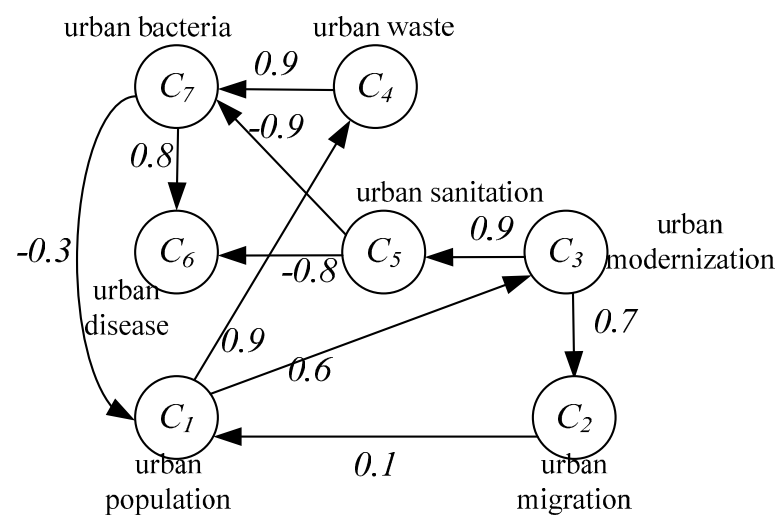

Figure 1. A fuzzy cognitive mapstructure. 


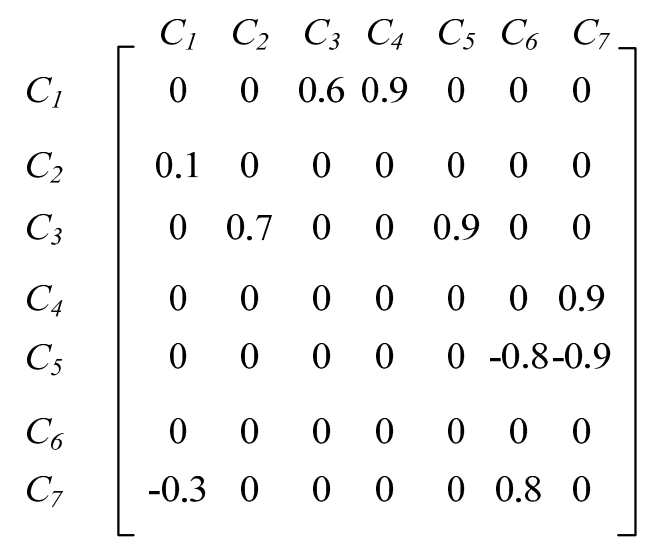

Figure 2. Weight matrix of the FCM.

$A$ is state space of the FCM. At $t$ iteration, the state of the FCM is a state vector $A(t)=\left(A_{1}(t), A_{2}(t)\right.$, $\left.\ldots, A_{n}(t)\right)$, whereeach concept node $C_{i}$ has a state value $A_{i}(t)$.

The state value of $C_{j}$ at $t+1$ step can be deduced by a sigmoid function $f(x)$ shown as Equation (1).

$$
A_{i}(t+1)=\mathrm{f}\left(\sum_{j} A_{j}(t) w_{j i}\right)
$$

The functioncan bea bivalent function as in Equation (2), a trivalent function as in Equation (3), or a logistic function as in Equation (4). The state value of a node at $t+1$ iteration is a transformation function of states in a previous time of other nodesdirectly associated with the node and weight matrix of the FCM. Accordingly, each node state of the system can be obtainedby the inference mechanism.

$$
\begin{gathered}
\mathrm{f}(x)= \begin{cases}1 & x>T \\
0 & x \leqslant T\end{cases} \\
\mathrm{f}(x)=\left\{\begin{array}{rl}
1 & x \geqslant T \\
0 & -T<x<T \\
-1 & x \leqslant-T
\end{array}\right. \\
\mathrm{f}(x)=\frac{1}{1+e^{-c x}}
\end{gathered}
$$

\section{Steady States of the Fuzzy Cognitive Map}

Definition 1. Fixed point is a kind of steady state where theultimate state of the FCM is a unique state vector, that is $A(t+1)=A(t)$.

Definition 2. Limited cycle is a kind of steady state where theFCM settles down with a state vector repeating in the form $A(t) \rightarrow \ldots \rightarrow A(i) \rightarrow \ldots \rightarrow A(t)$, that is $A(t+K)=A(t)(K>1)$.

The following research in the paper is aimed at a kind of simple FCM, which has a bivalent function, so each node state is 1 or 0 .

Inference 1. The FCM must settle down with fixed point or limited cycle.

If the FCM transforms to a fixed point, all concepts in the system are positive or negative. In other words, if the FCM transformsto a 0 fixed point that is the vector $[0,0, \ldots, 0]$, the systems arenegative; if the FCM transformstoa 1 fixed point that is the vector $[1,1, \ldots, 1]$, the concepts arepositive.

If the FCM transforms to a limited cycle, the system will return to the initial state. The recurrence shows that the system is relatively positive.

Definition 3. The number of iterations of steady state is the number of steps from the initial state to the ultimate state in the process of the FCM transforming to a steady state. 
Definition 4. The number of state transformations of steady state is the total number of nodeswhose state changesin the process of the FCM transforming to a steady state.

For example, in Figure 3 the number of iterations of the limited cycle is three, and the number of state transformations of the limited cycle is six (the sum of one, two and three).

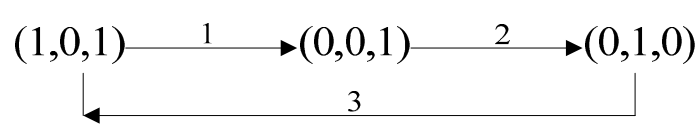

Figure 3. A limited cycle from initial state $(1,0,1)$

As is known, in next step the changes of states are caused by node states in the step, threshold function and weights of the FCM. The threshold $T$ of the sigmoid function in the FCM indicates an alarm value or an evaluation standard of the system. The weights of the FCM are on behalf of the current status of the system. The paper focuses on a simple FCM with bivalent function. After a number of iterations, the FCM state will transform to a steady state.

\subsection{Fixed Point of the FCM}

Inference 2. If initial states of all nodes in the FCM are zero and $T=0$, it must transform toa zero fixed point.

Inference 3. If the threshold $T>\max \left(\sum w_{j i}\right)$ is in the $\mathrm{FCM}$, it must transform to a zero fixed point.

Inference 4. If the threshold $T<\min \left(\sum_{j}^{j} w_{j i}\right)$ is in the FCM, it must arrive toa 1 fixed point.

Inference 5. If only one node state ofthe FCM changes at $t$ step, then the state of the node must be the same with thatat the $t+1$ step.

Inference 6. The number of iterations of steady state must be less than or equal to the number of nodes, which is less than or equal to the number of state transformations of steady state in the process of the FCM transforming to fixed point.

For example, the initial state is [1, 1, 1], the weight matrix is shown in Equation (5) and $T=0$.

$$
W=\left[\begin{array}{ccc}
0 & -0.4 & -0.3 \\
0.4 & 0 & -0.1 \\
-0.3 & 0.5 & 0
\end{array}\right]
$$

The process ofstate transformations of steady state is shown in Figure 4. In the transformation, only one node state changes in each iteration.

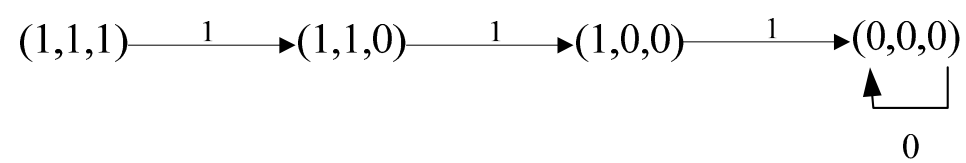

Figure 4. The longest length in the FCM.

In the longest length case, the length or the number of iterations of the fixed point is equal to the number of nodes, which is equal to the number of state transformations.

\subsection{Limited Cycle of the FCM}

Definition 5. The length of a limited cycle is the number of iterations in the process of the FCM transforming to the initial state in the limited cycle.

Inference 7. The limited cycle of the FCM must be formed by even numbers of state transformations in the cycle. 
Inference 8. The length of a limited cycle must be less than or equal to the number of state transformations in the process of the FCM transforming to the initial state in the limited cycle.

Inference 9. The number of state transformations is twice the number of nodes whose states change in the FCM arriving ata limited cycle.

The proofs of Inference 1-Inference 9 are shown in Appendix.

\section{The Application in Three-Rivers Ecosystem}

The Three-Rivers ecological zone, in the southern part of the Qinghai province of China, is the birthplace of the Yangtze River, Yellow River and Lancang River of China. It is China's largest and highest natural area, and is also the most sensitive natural environment and vulnerable region. Twenty-five percent of the Yangtze River water, $49 \%$ of the Yellow River water and $15 \%$ of theLancang River water are from thisregion. Thus, the situation of the Three-Rivers ecological system is the ecological guarantee of China's development, even tied with the ecological security of the neighboring countries. Because of the special geographical conditions, global climate change and the increasingly frequent effect of human economic activities, the contradictions in the Three-Rivers ecological system become more and more apparent, and mainly reflect in the relationships among the population, ecological environment and social development.

\subsection{FCM Modeling Three-Rivers EcosystemBased on NHL}

Accordingly, the FCM model of the Three-Rivers system is used to represent population, ecological environment, social development and their relationships, and to study the evolutions of the Three-Rivers ecosystem from the macro-level.

Therefore, the FCM model of the Three-Rivers ecosystem includes three concept nodes of population, ecological environment and social development, whose states are $\left(A_{1}, A_{2}, A_{3}\right)$ representing the evaluation value of population, ecological environment and social development, respectively. The association map is shown in a weight matrix in Equation (6).

$$
W=\left[\begin{array}{ccc}
0 & -0.7 & 0.3 \\
0.4 & 0 & 0.1 \\
-0.3 & 0.5 & 0
\end{array}\right]
$$

The weights are obtained by the NHL algorithm based on big data from the Internet of things and statistic database, which show current system conditions. NHL has two objective functions, as shown in Equations (7) and (8).

$$
\begin{gathered}
\operatorname{maximize} J=E\left[\sum A_{j}\right] \\
\left|A_{j}^{t}-A_{j}^{t+1}\right|<e
\end{gathered}
$$

The weight vector $w$ has to be limited to stabilize the learning rule in Equation (9), which generates the following nonlinear Hebbian learning rule in Equation (10).

$$
\begin{gathered}
\text { subject to }\|w\|=1 \\
w_{i j}(t+1)=\eta A_{j}\left(A_{i}-w_{i j}(t) A_{j}\right)
\end{gathered}
$$

The learning steps are as follows.

Step1: Calculate the evaluation values of population, ecological environment and social development based on large monitoring data and statistic data.

Step2: Initialize the weight matrix.

Step3: Calculate the objective function of the NHL.

Step 3.1: Adjust the weights by the NHL rules. 
Step 4: Continue until the weights satisfy the objective function of the NHL.

\subsection{Sustainability Analysis of theThree-Rivers Ecosystem Based on the FCM}

The bivalent function in Equation (2) is selected as the transformation function. Based on these, we obtain the following analysis results on the ecological system evolution.

4.2.1. Situation 1: If The Initial State is $(1,0,1)$ and The Threshold Value $T=0$, What May Happen in The System?

The initial state means that population is increasing and society is in development. The datum line $T$ of the evaluation is zero. The state value of each concept is computed at each step by Equations (1) and (2).

Thus, the process of system evolutionis shown in Figure 3.

There is only one state transformation of node $C_{1}$ from 1 to 0 in the first iteration. In the second step, there are two state transformations of $C_{2}$ and $C_{3}$, respectively. In the final iteration, the three nodes all are reversed. The number of iterations of the limited cycle is three. The number of state transformations of the limited cycle is six.It fully complies with the above rules from Inference 7-Inference 9 of the FCM.

It can be seen that the situation will first make the population decrease relatively, followed by an improvement of the environment and a slowdown in the social development. Finally, the recurrence represents that the population and social development are picked up again, and the relative destruction of the environment follows. The process represents a limited cycle that shows an implacable contradiction among population, ecological environment and social development. The Three-Rivers ecological system is in a relatively positive or sustainable evolution.

\subsubsection{Situation2: What Will Happen with Other Initial States?}

The FCM including three nodes has eight different states in total, where the process in Discussion 1 involves three FCM states. The four other states except for $(0,0,0)$ all form into a limited cycle.

The process of the transformations from states $(0,1,1)$ and $(1,1,1)$ is shown as a limited cycle in Figure 5.

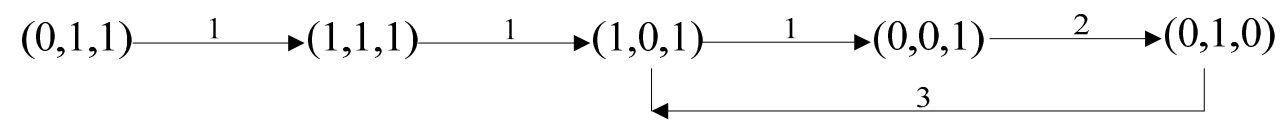

Figure 5. A state evolution from the states $(0,1,1)$ or $(1,1,1)$.

Fromstate $(1,0,0)$, the process is shown as a limited cycle in Figure 6.

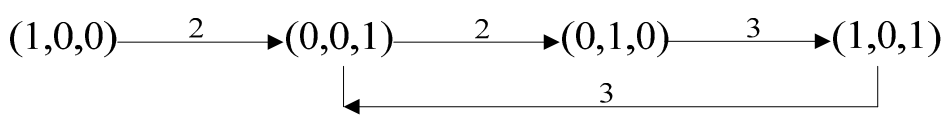

Figure 6. A state evolution from the state $(1,0,0)$.

From state $(1,1,0)$, the process is shown as a limited cycle in Figure 7.

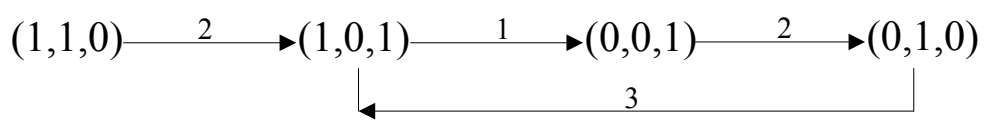

Figure 7. A state evolution from the state $(1,1,0)$. 
In actuality, the above three processes all include a similar limited cycle as in Situation 1. The transformations all comply with the above rules from Inference 7-Inference 9of the FCM. The three situations still are in sustainable evolution.

The state $(0,0,0)$ transforms toa 0 fixed point in accordance with Inference 2 . It means that when all concepts of the system are in down states, the system must be a downward or not sustainable trend unless there are positive energy incentives.

How to break this vicious cycle?

The method is to change the associated weights between the concepts through external intervention such as policy, law, etc.

\subsubsection{Situation3: If the Datum Line T of Evaluation Increases or Decreases, What Will Happen?}

When the initial state is $(1,0,1)$ and the threshold value increases to $T>0.4$, a 0 fixed point is formed according to Inference 3 .

The process of system state evolution at $T=0.5$ is shown as a fixed point in Figure 8 . It can be seen that when the threshold increases in given weights and initial state, the limited cycle in Figure 4 may change to a fixed point as in Figure 8.

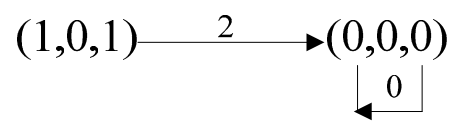

Figure 8. A state evolution from initial state $(1,0,1)$.

The initial state is $(1,0,1)$ and the threshold value $T=-0.1$ or -0.2 .

The process of the system state evolution is shown in Figure 9. The FCM still transforms to a limited cycle. It can be seen that when the threshold decreases in given weights and initial state, the number of iterations of the limited cycle in Figure 9 is more than it is in Figure 4.

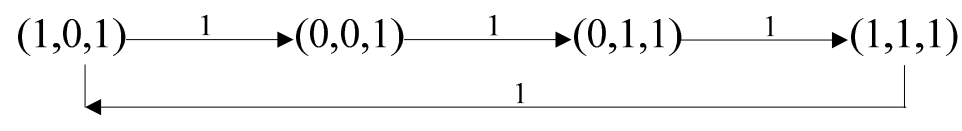

Figure 9. A limited cycle from initial state $(1,0,1)$.

When the threshold value decreases to $T<-0.2$, a 1 fixed point is formed according to Inference 4. The process of the system state evolution at $T=-0.3$ is shown in Figure 10. It can be seen that when the threshold decreases furtherin given weights and initial state, the limited cycle in Figure 4 may change to a fixed point as in Figure 10.

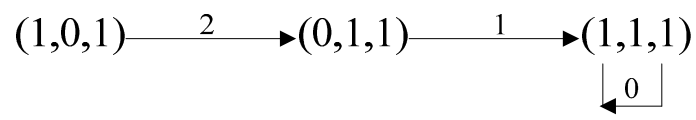

Figure 10. A state evolution from initial state $(1,0,1)$ to a fixed point.

In this situation, first the population is reduced and the environment is improved, followed by a relativeincrease of population. The FCM transforms to a 1 fixed point, where the relationship betweenpopulation, ecological environment and social development forms into a positive steady state or a sustainable system.

It means that standard $(T)$ plays a vital role in systemevolution. It can be seen that the FCMcan provide the basis for determining the standard. 


\subsubsection{Situation4: In the System, What Must Not Happen?}

If it goes against the inferencesof the FCM, the process will not happen.

Meanwhile, some state transformations in the current system may be excluded. For example, the situation in Figure 11 cannot happen at $T=0$. Although it complies with the rule of limited cycle or sustainability, withthis current threshold and relationships it is impossible.

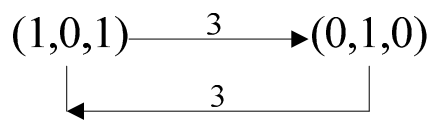

Figure 11. An impossible limited cycle in the system evolution.

\section{Conclusions}

In short, the steady states of FCM can reflect system evolution. Additionally, thesteady states of the FCM are related tonode states and state transformations, such as the initial states of the nodes, the state changes of a node in a moment, and the number of state transformations. Thus, the system evolution can be controlled efficiently according to the rules of steady states. In the application, the Three-Rivers ecosystem evolution is the direct result of the concept states and the relationships among them under the limit of the threshold. At the same time, the evolution of the Three-Rivers ecosystem has verified the theory of steady states of FCM, which can help to further analyze and control thesustainable evolution of the Three-Rivers ecosystem.

Acknowledgments: This research was supported by the Natural Science Foundation of Province (No. F2014508028), the Project of Beijing Social Science Foundation (No.15JDJGB028), the Project of Construction of Innovative Teams and Teacher Career Development for Universities and Colleges Under Beijing Municipality (No.IDHT20150507), and the Scientific Research Base Development Program of the Beijing Municipal Commission ofEducation (TJSHG201310028014).

Author Contributions: Zhen Peng performed the studies on FCM steady states and the analysis on Three-Rivers ecosystem. Zhen Peng, LifengWu and ZhenguoChen carried out experimental work. Zhen Peng prepared the manuscript.

Conflicts of Interest: The authors declare no conflict of interest.

\section{Appendix}

Inference 1. The FCM must settle down with fixed point or limited cycle.

Proof: The state space is finite in the FCM and each node state is 0 or 1 . Thus, the FCM must settle down with fixed point or limited cycle.

Inference 2. If initial states of all nodes in the FCM are zero and $T=0$, it must transform toa zero fixed point.

Proof: To any node $C_{j}, A_{j}(0)=0$ is known. According to Equations (1) and (2), whatever the FCM weight values are, the threshold $T=0$, and next states of the FCM are certainly all zero. Thus, Inference 2 is true.

Inference 3. If the threshold $T>\max \left(\sum_{j} w_{j i}\right)$ is in the $\mathrm{FCM}$, it must transform to a zero fixed point.

Proof: In Equation (1), $A_{j}$ is 0 or 1.

If $T>\max \left(\sum_{j} w_{j i}\right)$, then $T>\sum_{j} A_{j}(t) w_{j i}$. Accordingly, in the FCM with bivalent function $A_{i}(t+1)$ $=\mathrm{f}\left(\sum_{j} A_{j}(t) w_{j i}\right)=0$. Thus, Inference 3 is true.

Inference 4. If the threshold $T<\min \left(\sum_{j} w_{j i}\right)$ is in the $\mathrm{FCM}$, it must arrive at a 1 fixed point.

Proof: It is similar to the proof of Inference 3.

Inference 5. If only one node state ofthe FCM changes at $t$ step, then the state of the node must be the same at that at the $t+1$ step. 
Proof: Assume $C_{i}$ is the node. At $t$ step, eachnode $C_{j}(\mathrm{j} \neq \mathrm{i})$ is $A_{j}(t-1)=\mathrm{A}_{\mathrm{j}}(t)$. Then, at $t+1$ step, by Equation (1): $A_{i}(t+1)=f\left(\sum_{j} A_{j}(t) w_{j i}\right)=f\left(\sum_{j} A_{j}(t-1) w_{j i}\right)=A_{i}(t)$. Thus, Inference 5 is true.

Inference 6. The number of iterations of steady state must be less than or equal to the number of nodes, which less than or equal to the number of state transformations of steady state in the process of the FCM transforming to a fixed point.

Proof: The number of state transformationsof a node is less than or equal to two in the FCM transforming toa fixed point. The longest length happens when only one node state changes in each iteration.

In that case, the length or the number of iterations of the fixed point is equal to the number of nodes, which is equal to the number of state transformations.

If more than one node states change in an iteration, the length or the number of iterations of the fixed point must be less than the number of nodes, which is less than the number of state transformations. Thus, Inference 6 is true.

Inference 7. The limited cycle of the FCM must be formed by even numbers of state transformations in the cycle.

Proof: (reduction to absurdity) If the FCM passes odd transformations in the limited cycle, there is at least one node that passes odd numbers of state transformations. Thus, it is impossible that a node returns to the initial state through odd numbers of transformations. Nevertheless, the total number of transformations must be even. Thus, Inference 7 is true.

Inference 8 . The length of a limited cycle must be less than or equal to the number of state transformations in the process of the FCM transforming to the initial state in the limited cycle.

Proof: A node must go through two transformations or no transformation in the process of the FCM transforming to the initial state in thelimited cycle. If there is only one node state changing in each iteration, the length of the limited cycle must be equal to the number of state transformations of the FCM. If there is more than one node whose state changes in an iteration, the length of the limited cycle must be less than the number of state transformations of the FCM. Thus, Inference 8 is true.

Inference 9. The number of state transformations is twice of the number of nodes whose states change in the FCM arriving to a limited cycle.

Proof: A node must go through two transformations or no transformation in the FCM arriving at a limited cycle. The number of state transformations is twice the number of nodes whose states are changed in the FCM arriving ata limited cycle. Thus, Inference 9 is true.

\section{References}

1. Kosko, B. Fuzzy cognitive maps. Int. J. Man-Mach. Stud. 1986, 24, 65-75. [CrossRef]

2. Stylios, C.D.; Gmumpca, P.P. Fuzzy cognitive maps: A soft computing technique for intelligent contro1. In Proceedings of the IEEE International Symposium on Intelligent Control, Rio Patras, Greece, 17-19 July 2000; pp. 97-102.

3. Pelaez, C.E.; Bowles, J.B. Using fuzzy cognitive maps as a system model for failure modes and effects analysis. Inf. Sci. 1996, 88, 177-199.

4. Liu, Z.Q. Fuzzy cognitive maps in GIS data analysis. Soft Comput. 2003, 7, 394-401. [CrossRef]

5. Mago, V.K.; Bakker, L.; Papageorgiou, E.I.; Alimadad, A.; Borwein, P.; Dabbaghian, V. Fuzzy cognitive maps and cellular automata: An evolutionary approach for social systems modeling. Appl. Soft Comput. 2012, 12, 3771-3784. [CrossRef]

6. Froelich, W.; Papageorgiou, E.I.; Samarinas, M.; Skriapas, K. Application of evolutionary fuzzy cognitive maps to the long-term prediction of prostate cancer. Appl. Soft Comput. 2012, 12, 3810-3817. [CrossRef]

7. Buche, C.; Chevaillier, P.; Nedelec, A.; Parenthoen, M.; Tisseau, J. Fuzzy cognitive maps for the simulation of individual adaptive behaviors. Comput. Anim. Virtual Worlds 2010, 21, 573-587. [CrossRef]

8. Gray, S.A.; Gray, S.; Cox, L.J.; Henly-Shepard, S. Mental modeler: A fuzzy-logic cognitive mapping modeling tool for adaptive environmental management. In Proceedings of the 46th Hawaii International Conference on System Sciences, Wailea, HI, USA, 7-10 January 2013; pp. 965-973. 
9. Papakostas, G.A.; Koulouriotis, D.E.; Polydoros, A.S.; Tourassis, V.D. Towards Hebbian learning of fuzzy cognitive maps in pattern classification problems. Expert Syst. Appl. 2012, 39, 10620-10629. [CrossRef]

10. Stach, W.; Kurgan, L.; Pedrycz, W. A divide and conquer method for learning large fuzzy cognitive maps. Fuzzy Sets Syst. 2010, 161, 2515-2532. [CrossRef]

11. Ahmadi, S.; Forouzideh, N.; Alizadeh, S.; Papageorgiou, E.I. Learning Fuzzy Cognitive Maps using Imperialist Competitive Algorithm. Neural Comput. Appl. 2015, 26, 1333-1354. [CrossRef]

12. Luo, X.; Yao, E. The reasoning mechanism of fuzzy cognitive maps. In Proceedings of the First International Conference on Semantics, Knowledge and Grid (SKG'05), Beijing, China, 27-29 November 2005; pp. 27-29.

13. Djam, X.Y.; Mishra, A.K. Fuzzy cognitive map based approach for teachers' performance evaluation. Pac. J. Sci. Technol. 2013, 14, 176-181.

14. Salmeron, J.L. Fuzzy cognitive maps for artificial emotions forecasting. Appl. Soft Comput. 2012, 12, 3704-3710. [CrossRef]

15. Lee, S.; Yang, J.; Han, J. Development of a decision making system for selection of dental implant abutments based on the fuzzy cognitive map. Expert Syst. Appl. 2012, 39, 11564-11575. [CrossRef]

16. Kandasamy, W.B.V.; Smarandache, F. Fuzzy Cognitive Maps and Neutrosophic Cognitive Maps; Xiquan Pub.: Phoenix, AZ, USA, 2003.

17. Bevilacqua, M.; Ciarapica, F.E.; Mazzuto, G. Analysis of injury events with fuzzy cognitive maps. J. Loss Prev. Process Ind. 2012, 25, 677-685. [CrossRef]

18. Knighta, C.J.K.; Lloydb, D.J.B.; Penna, A.S. Linear and sigmoidal fuzzy cognitive maps: An analysis of fixed points. Appl. Soft Comput. 2014, 15, 193-202. [CrossRef]

(C) 2016 by the authors; licensee MDPI, Basel, Switzerland. This article is an open access article distributed under the terms and conditions of the Creative Commons by Attribution (CC-BY) license (http://creativecommons.org/licenses/by/4.0/). 